\title{
Polymer-conjugated guanidine is a potentially useful anti-tumor agent
}

\author{
LENNART MEURLING, MARCELA MÁRQUEZ, STEN NILSSON and ANDERS R. HOLMBERG \\ Department of Oncology and Pathology, Karolinska Institute, SE-171 76 Stockholm, Sweden
}

Received January 26, 2009; Accepted March 13, 2009

DOI: 10.3892/ijo_00000338

\begin{abstract}
Guanidine compounds have important biochemical properties. Aminoguanidine, as an example, is an anti-oxidant, a nitric oxide synthase inhibitor (NOS) which prevents nitric oxide formation, and an inhibitor of advanced glycosylation end products (AGEs). As an anti-oxidant, aminoguanidine may affect the formation of atherosclerotic lesions through protection from LDL oxidation. Inhibition of AGEs could have a preventive effect on the tissue damage caused by diabetes where AGEs are considered to be an important factor. The role of NO in cancer is complex and not fully understood, but it may have influence on growth and progression. In this study, the tumor growth inhibitory effect of conjugated guanidine (i.e. a polyguanidine) was investigated. The effect on tumor cell growth was studied in cultures of prostate, breast, bladder and renal cell cancer, and a fluorometric cytotoxicity assay was performed. Guanidine conjugates were prepared by reacting aminoguanidine or agmatine with periodate oxidized dextran followed by reductive amination. The cytotoxic effect was compared with an anthracycline (adriamycin). The dextran-guanidine conjugates were cytotoxic at low micromolar concentrations, and the dextranaminoguanidine conjugate (GDC) had the highest efficacy, being more efficient than adriamycin, in all of the tested tumor cell lines. Breast and prostate cancer cells were the most sensitive. At $0.5 \mu \mathrm{M}$, GDC killed $>95 \%$ of the breast cancer cells compared to $25 \%$ for Adriamycine. In prostate cancer cells, GDC killed $\sim 55 \%$ of the cells at $0.1 \mu \mathrm{M}$ and $100 \%$ of the cells at $0.5 \mu \mathrm{M}$ compared to $\sim 22$ and $\sim 62 \%$, respectively, for adriamycin. Unconjugated aminoguanidine and agmatine did not seem to affect tumor cell growth even at high concentrations (mM). Polymer- conjugated guanidine is a potentially useful template for the construction of therapeutic tumor targeting cytotoxic agents.
\end{abstract}

Correspondence to: Dr Anders R. Holmberg, Urologic Oncology Group, CCK R8/3, Karolinska University Hospital, Karolinska Institute, SE-171 76 Stockholm, Sweden

E-mail: arh@telia.com

Key words: dextran, guanidine, conjugates, anti-tumor

\section{Introduction}

The guanidine group is present in a large number of biologically active compounds including drugs and drug candidates. Applications for such compounds include prevention of cardiovascular damage, antihistamine, antiinflammatory, antidiabetic, antibacterial/antiviral, and as anticancer drugs. The guanidine sidegroup contributes to the variety of applications of these compounds deriving from its ability to recognize receptors by noncovalent interactions, e.g. hydrogen-bonding and electrostatic binding. (1). Examples of guanidine compounds are aminoguanidine, metformin, arginine and agmatine.

Aminoguanidine is an anti-oxidant and an inhibitor of advanced glycosylation end products (AGEs), and its properties may slow the formation of atherosclerotic lesions and cardiovascular damage through protection from LDL oxidation and the tissue damaging effects of AGEs. In fact, glycosylation of proteins (the 'Maillard reaction') is one of the most important causes of aging/degeneration, perhaps equally important as the process of oxidation. The product of the Maillard reaction (an 'Amadori product') may then crosslink with other proteins resulting in irreversible bonding. AGE formation happens as a result of normal aging and is accelerated during hyperglycemic states such as diabetes. Aminoguanidine is currently supplied as a non-prescription drug in the US, and its role in the treatment of disease is being investigated in clinical research. Metformin is a bi-guanidine and the most commonly prescribed drug for type 2 diabetes. It improves hyperglycemia primarily through suppression of hepatic glucose production and can lower the occurrence of cardiovascular damage (2-6).

Arginine has a guanidine sidegroup and is a semi-essential amino acid with multiple key roles in cellular metabolism. It is an important precursor in the biosynthesis of a number of substances. Arginine is essential in cellular growth and recovery after injury and stimulates the release of several hormones (7). It is the sole precursor for nitric oxide (NO), a messenger molecule with multiple functions. The exact role of NO in cancer is not fully understood but may influence tumor initiation, promotion and progression (8). In contrast, aminoguanidine is a nitric oxide synthase inhibitor (NOS) which prevents NO formation (9).

Additionally, aminoguanidine is a chaotrope, i.e. it can disrupt the three-dimensional structure of macromolecules and DNA/RNA (denaturation). A high concentration is required to have this effect. 
Agmatine is the decarboxylation product of arginine and is an inducer of antizyme and in this way inhibits cell growth (10). Antizyme inhibits the activity of ornithine decarboxylase (ODC), a rate limiting enzyme in the polyamine biosynthetic pathway. A relatively high concentration of agmatine is required $(\mathrm{mM})$.

Guanidine alkaloids deriving from natural marine sponges and their synthetic derivatives have been found to have significant anticancer/antiviral properties (11).

In perspective of these highly significant and diverse properties, the present study investigated the antiproliferative/cytotoxic properties of polyguanidine conjugates on tumor cell cultures. For this purpose, guanidines (aminoguanidine or agmatine) were conjugated to a carbohydrate polymer (dextran) in high yield. The growth inhibitory effect was tested on prostate, breast, bladder and renal cell cancer cell lines. A conventional chemotherapeutic drug (adriamycin) was used for comparison at equimolar concentrations.

\section{Materials and methods}

Dextran 70 PhEUR (Pharmacosmos AS, Denmark) was used as a conjugate backbone. Sodium meta-periodate (Merck AG, Darmstadt Germany) was used for dextran oxidation (activation). Aminoguanidine and agmatine (Sigma-Aldrich, Sweden) were used for the coupling. Sodium cyanoborohydride (Chemicon, Stockholm, Sweden) was used for reductive amination. NAP-5 and PD-10 disposable Sephadex G-25 columns were used for separation and purification (Pharmacia Amersham Biotech AB). Adriamycin was from Sigma-Aldrich, Sweden.

Activation. Vicinal hydroxyl groups in the dextran molecule were oxidized to dialdehydes by means of periodate (oxidation) at an acidic $\mathrm{pH}$ (12). The oxidation was monitored by following the absorption of the periodate ion at $315 \mathrm{~nm}$. At this wavelength, periodate has a substantial absorption, while iodate does not interfere. It was found that the reaction strictly follows first order kinetics with a half-life of $5 \mathrm{~min}$. This means that after $30 \mathrm{~min} \sim 1.5 \%$ of the initial periodate remains. Adding an equimolar amount of etylenglycol and running the reaction for an additional 15 min assures that all periodate reacts to iodate. This is crucial since the remaining periodate oxidizes the amine in the coupling step, giving colored by-products. Since iodate does not interfere in the coupling step, purification is not necessary (G-25 separation) before the coupling step.

Dextran $(0.3 \mathrm{~g})(4.3 \mu \mathrm{mol})$ was dissolved in $10 \mathrm{ml}$ of deionised water. Concentrated sulphuric acid (50 $\mu \mathrm{l})$ was added, followed by $0.240 \mathrm{~g}(1.12 \mathrm{mmol})$ of sodium periodate. The reaction mixture was stirred in the dark at room temperature for $30 \mathrm{~min}$. Then $65 \mu \mathrm{l}$ of etylenglycol was added, and the mixture was incubated for $15 \mathrm{~min}$. The oxidized dextran was used directly in the conjugation step or frozen in aliquots for later use.

Conjugation. Aminoguanidine ( $80 \mathrm{mg}$ ) (or $80 \mathrm{mg}$ agmatine) $+5 \mathrm{mg}$ sodium cyanoborohydride were mixed with $15 \mathrm{mg}$ of activated dextran, all in $1 \mathrm{ml}$ of $0.25 \mathrm{M}$ sodium acetate at $\mathrm{pH}$ 6.5. The solution was incubated by gentle shaking for $4 \mathrm{~h}$ (in the dark at room temperature). After incubation, the solution was purified on a PD-10 column, using saline as an eluent.

Determination of the conjugation yield. The conjugation yield, i.e. the number of guanidine groups coupled to the dextran backbone, was determined using two methods: total nitrogen content by elemental analysis or with a spectrophotometric method.

Elemental analysis. Total nitrogen content was determined by LSM Group, Uppsala, Sweden.

Spectrophotometric method. Determination of guanidine residues, and thus indirectly the arginine content in proteins, is most frequently conducted with the Sakaguchi procedure (13). Many modifications of this method have been published $(14,15)$. Most methods have been found to give reliable and reproducible results for the arginine content in proteins. All of these methods have, however, one problem in common, i.e. finding a suitable standard. It has been found that the results vary depending on the standard.

In the present study, the Sakaguchi method for the determination of the guanidine content was employed. The most reliable results were obtained with the Van Pilsum modification (16). A highly substituted dextran preparation was used as a standard when the guanidine content was determined by elemental analysis.

All reagents were from Sigma-Aldrich AB, Stockholm, Sweden and of pro-analysis quality. Absorption measurements were made on a spectrophotometer at $515 \mathrm{~nm}$ (GE Biotech, Uppsala, Sweden).

Reactants. Thymidine (200 mg) was dissolved in $10 \mathrm{ml}$ of $10 \%$ sodium hydroxide (A). 1-Naphtol (40 mg) was dissolved in $100 \mathrm{ml}$ of absolute ethanol (B). Sodium hypochlorite (1\%) was prepared from commercial $10 \%$ hypochlorite solution (C). Sodium thiosulphate $(2 \mathrm{~g})$ was dissolved in $100 \mathrm{ml}$ of water (D).

Procedure. One hundred microliters of guanidinemodified dextran solution was pipetted into a test tube and diluted to $1 \mathrm{ml}$ and cooled on ice. Five hundred microliters of an ice-cooled 1/1 mixture of A and B was added, followed by $200 \mu 1$ of hypochlorite solution $\mathrm{C}$. The mixture was rapidly stirred by short vortexing and allowed to react for exactly $1 \mathrm{~min}$. The oxidation was terminated by the addition of $200 \mu \mathrm{l}$ thiosulphate solution $\mathrm{D}$, and the absorption was determined at $515 \mathrm{~nm}$ against a suitable blank. The red-brown color was stable at $0^{\circ} \mathrm{C}$ for at least $15 \mathrm{~min}$, but faded rapidly when warmed.

Fluorimetric cytotoxicity assay (FMCA). The assay was performed as described by Larsson and Nygren (17). The following cell lines were used: urinary bladder carcinoma RT4, breast carcinoma MDA 453, prostate adenocarcinoma PC-3 and renal cell cancer A498 (all from ATTC, Manassas, VA, USA).

Briefly, 10,000 cells/well were seeded onto 96-well microtiter plates (Falcon; Becton Dickinson, Meylan, France). Adriamycin and dextran-guanidine conjugate were added at equimolar concentrations $(0.1-40 \mu \mathrm{M})$. The control wells were furnished with the same amount of PBS. After a $72 \mathrm{~h}$ incubation, the microtiter plates were centrifuged ( $200 \mathrm{x} \mathrm{g}$ 


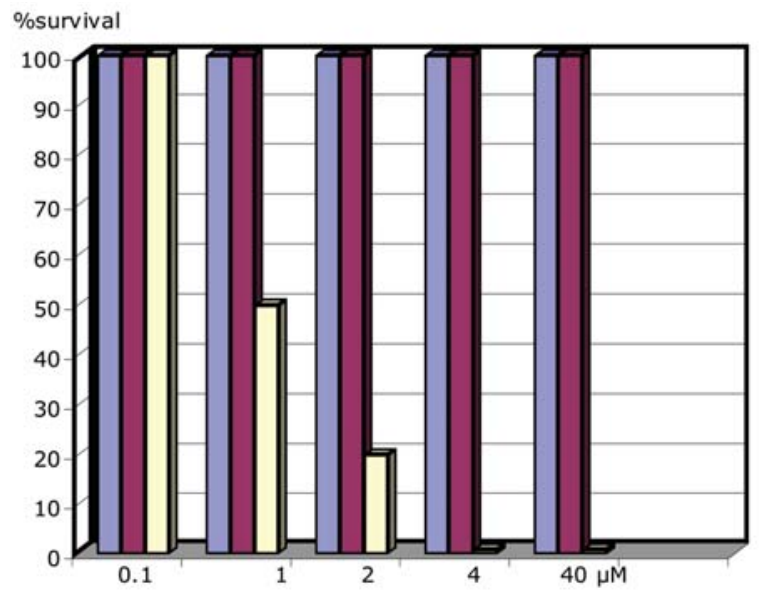

Figure 1. $\mathrm{y}$-axis, \% growth inhibition; $\mathrm{x}$-axis, ligand concentration. Blue, aminoguanidine; yellow, anthracycline; red, dextran. Data represent mean values, $\mathrm{n}=3, \mathrm{cv}<5 \%$ (RT4 cell line).

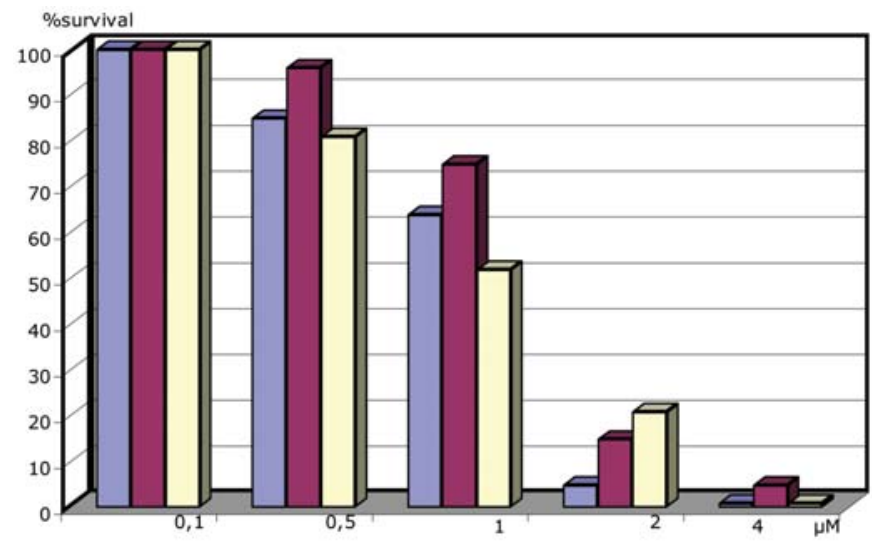

Figure 2. y-axis, \% growth inhibition; $\mathrm{x}$-axis, ligand concentration. Blue, aminoguanidine-dextran; red, agmatine-dextran; yellow, anthracycline. Data represent mean values, $\mathrm{n}=3, \mathrm{cv}<5 \%$ (RT4 cell line).

for $3 \mathrm{~min}$ ), and the medium was removed by flicking the plates. The cells were washed in PBS. Fluorescein diacetate (FDA, Sigma) was dissolved in DMSO and kept frozen at $-20^{\circ} \mathrm{C}$ as a stock solution $(10 \mathrm{mg} / \mathrm{ml})$. The FDA was diluted in PBS at a concentration of $10 \mu \mathrm{g} / \mathrm{ml}$, and $200 \mu \mathrm{l}$ was added to each well. The plates were then incubated for $30 \mathrm{~min}$ at $37^{\circ} \mathrm{C}$. A 96-well scanning fluorometer (Fluoroscan 2, Labsystems, Helsinki, Finland) was used to count the emitted fluorescence. Data were transferred to a PC, and the results were calculated.

\section{Results}

Free, i.e. unconjugated, dextran and guanidine compound had no effect on the growth of the tumor cells (Fig. 1, RT4 bladder tumor cell line). Concentrations even at $\mathrm{mM}$ had no effect (data not shown).

When the guanidine compounds were conjugated to the dextran polymer, the resulting conjugate had a strong growth inhibitory effect on the tumor cell lines (Figs. 2-5). The conjugate with aminoguanidine had a higher tumor cell-

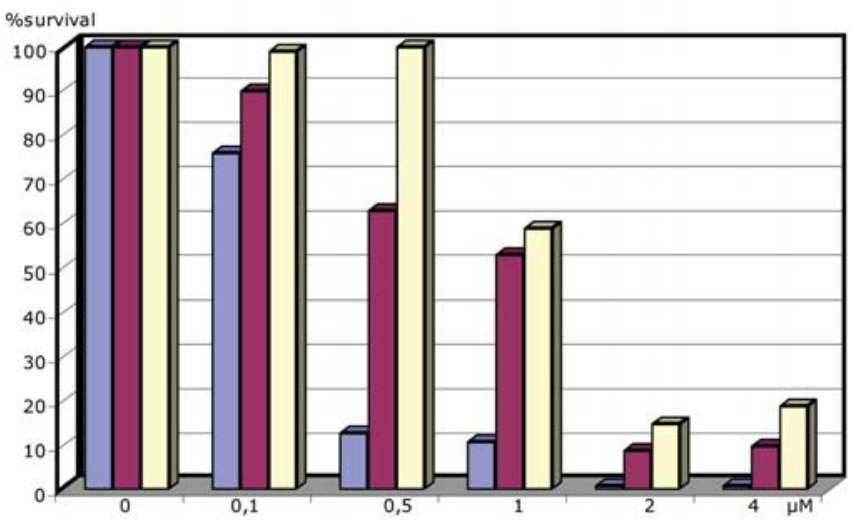

Figure 3. y-axis, $\%$ growth inhibition; $\mathrm{x}$-axis, ligand concentration. Blue, aminoguanidin-dextran; red, agmatine-dextran; yellow, anthracycline. Data represent mean values, $\mathrm{n}=3, \mathrm{cv}<5 \%$ (A498 cell line).

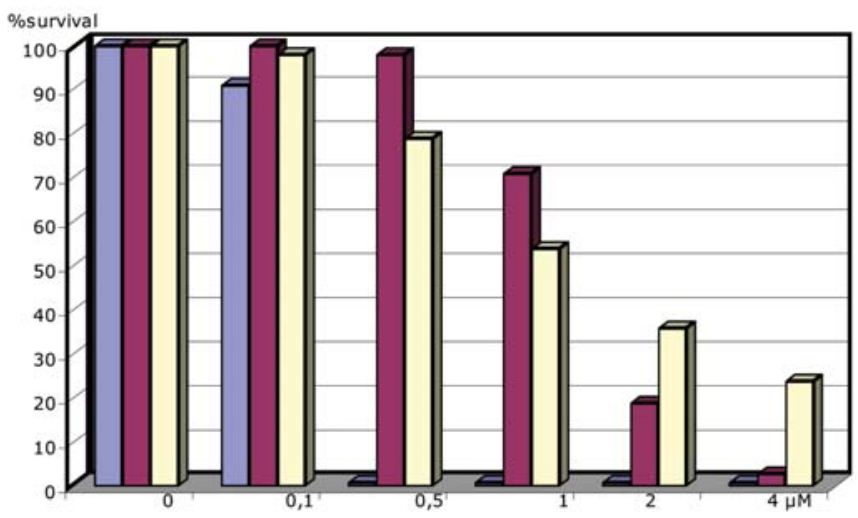

Figure 4. y-axis, $\%$ growth inhibition; $\mathrm{x}$-axis, ligand concentration. Blue, aminoguanidin-dextran; red, agmatine-dextran; yellow, anthracycline. Data represent mean values, $\mathrm{n}=3, \mathrm{cv}<5 \%$ (MDA453 cell line).

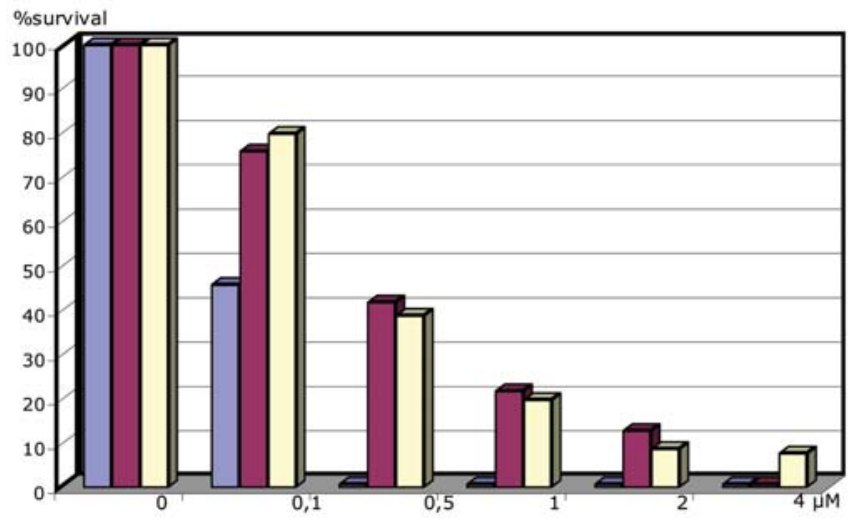

Figure 5. y-axis, \% growth inhibition; $\mathrm{x}$-axis, ligand concentration. Blue, aminoguanidin-dextran; red, agmatine-dextran; yellow, anthracycline. Data represent mean values, $\mathrm{n}=3, \mathrm{cv}<5 \%$ (PC3 cell line).

killing efficacy than the corresponding agmatine conjugate. Breast and prostate cancer cells were the most sensitive with few cells surviving at submicromolar concentrations. A high substitution of guanidine was required to obtain the strong 
growth inhibitory effect, i.e. $30-40 \%$ of the glucose residues substituted with guanidine sidegroups. A lower substitution decreased the tumor cell-killing efficacy. Substitution higher than $\sim 40 \%$ decreased the solubility of the conjugate, and precipitation occurred.

\section{Discussion}

In the present study, an anthracycline (adriamycin) was used as a positive control in the cytotoxicity assay. Anthracycline antibiotics are a class of drugs commonly used to treat several different types of cancers but never for the treatment of infections due to their toxicity, particularly cardiotoxicity (18). Regarding their mechanisms of action, the primary is the ability to induce genetic damage and cell death through DNA intercalation. Other actions include induction of apoptosis, free radical formation and lipid peroxidation. However, it is not clear whether interaction with DNA is necessary for apoptosis signaling $(19,20)$. One recent notable observation is the interaction between the human epidermal growth factor receptor family (HER) and anthracycline activity (21).

One characteristic of malignant growth is altered metabolism. The activation of signaling which stimulates cell growth has an extensive impact on anabolic metabolism. This fact in addition to its multiple consequences have been described as 'cancer's Achilles' heel' since it can be used in various ways as a target for therapeutic interventions (22).

To elucidate the present results, two mechanisms connected to tumor growth will be discussed, the polyamine uptake system and the $\mathrm{y}^{+}$cationic amino acid uptake system. They have similar transport characteristics but are distinct systems.

Polyamines are strongly cationic compounds which enter tumor cells with high effectiveness through a specific uptake system. The exact nature of this mechanism is still not yet completely defined (23). Polyamines are essential for tumor growth, and uptake is correlated with proliferation, i.e. high proliferation results in increased polyamine transmembrane transport. Similarly, arginine utilizing the $\mathrm{y}^{+}$system is conditionally essential during proliferation having numerous roles in cellular metabolism. The polyamine uptake system and polyamine synthesis are a target for tumor therapy, and many drugs focusing on biosynthesis enzymes, catabolic enzymes, DNA/RNA proteins and regulatory control systems have been developed (24). As an example of a polyaminecytotoxic conjugate utilizing this uptake system and the polyamine affinity to DNA, Holley and co-workers targeted DNA with a chlorambucil-spermidine conjugate (25).

The guanidine-dextran conjugate (GDC) is a glucose polymer with $>100$ positively charged guanidine sidegroups, i.e. a strongly cationic polyamine. Due to its resemblance with polyamines, and having the same sidegroup as arginine, it is reasonable to assume that GDC enters the cell employing the polyamine uptake system and possibly also through the $\mathrm{y}^{+}$ system.

Once inside the tumor cell there are several alternatives for explaining the apparent cytotoxicity of GDC. In fact, analogous to polyamines, the polycationic polymer can bind to numerous intracellular macromolecular structures, e.g. DNA, RNA, a variety of proteins and enzymes, membrane proteins and many small anionically charged phosphorylated molecules in the cytoplasm and nucleus (24). Besides the multiple possibilities for GDC to react and interfere, it might have choatropic effects disrupting the three dimensional structure of vital constitutions in the cytoplasm (26). It is plausible that the multi-cationic GDC molecule reacts with the multi-anionic polyphosphorylated DNA causing lethal damage. It is generally accepted that electrostatic interaction is one of the most important determinants of binding affinity between ligands and their targets, specific as well as nonspecific. Electrostatics plays a key role in defining the mechanisms of protein-protein complex formation, molecular recognitions, thermal stabilities, conformational adaptabilities and protein movements. For example, high affinity antibodies have higher, and stronger, electrostatic interactions with their antigens and rigid parts of proteins have higher and stronger electrostatic interactions.

The polyguanidine compound described in this study most probably employs cellular uptake systems connected to the anabolic stage of the proliferating tumor cells, in this manner gaining a certain tumor cell specificity. Its very likely that its cytotoxic effect is generated by electrostatics that eventually cause the toxicity. Further studies should reveal the detailed mechanism of the toxicity. However, owing to the many possible interactions, several components are most probably involved.

GDC may have many applications for therapeutic tumor targeting, with or without additional constituents. At present, GDC is being developed for the treatment of bone metastasis in advanced prostate cancer. The results will be reported in due course.

\section{Acknowledgements}

This study was supported by The Cancer Society in Stockholm; The King Gustav V Jubilee Fund, Stockholm; The Swedish Cancer Society; and Mr. Svante Wadman, Stockholm.

\section{References}

1. Nilsson BL and Overman LE: Concise synthesis of guanidinecontaining heterocycles using the Biginelli reaction. J Org Chem 71: 7706-7714, 2006.

2. Thornalley PJ: Use of aminoguanidine (Pimagedine) to prevent the formation of advanced glycation end products. Arch Biochem Biophys 419: 31-40, 2003.

3. Sell DR, Nelson JF and Monnier VM: Effect of chronic aminoguanidine treatment on age-related glycation, glycoxidation, and collagen cross-linking in the Fisher 344 rat. J Gerontol Biol Sci 56: 405-411, 2001.

4. Scaccini C, Chiesa G and Jialal I: A critical assessment of the effects of aminoguanidine and ascorbate on the oxidative modification of LDL: evidence for interference with some assays of lipoprotein oxidation by aminoguanidine. J Lipid Res 35: 1085-1092, 1994.

5. Dominiczak MH: Obesity, glucose intolerance and diabetes and their links to cardiovascular disease. Implications for laboratory medicine. Clin Chem Lab Med 41: 1266-1278, 2003.

6. Kilo C, Mezitis N, Jain R, Mersey J, McGill J and Raskin P: Starting patients with type 2 diabetes on insulin therapy using once-daily injections of biphasic insulin aspart 70/30, biphasic human insulin 70/30, or NPH insulin in combination with metformin. J Diabetes Complications 17: 307-313, 2003.

7. Lind DS: Arginine and cancer. Am Soc Nutr Sci J Nutr 134: 2837-2841, 2004 
8. Wink DA, Vodovotz Y, Laval J, Laval F, Dewhirst MW and Mitchell JB: The multifaceted roles of nitric oxide in cancer. Carcinogenesis 19: 711-721, 1998.

9. Nilsson BO: Biological effects of aminoguanidine: an update. Inflamm Res 48: 509-515, 1999.

10. Satriano J, Matsufuji S, Murakami Y, et al: Agmatine suppresses proliferation by frameshift induction of antizyme and attenuation of cellular polyamine levels. J Biol Chem 273: 15313-15316, 1998.

11. Berlinck RG: Natural guanidine derivatives. Nat Prod Rep 19: 617-649, 2002.

12. Matsunaga K, Kazumichi H, Imamura T, Fujioka T, Takata J and Karube Y: Technetium labelling of dextran incorporating cysteamine as a ligand. Nucl Med Biol 32: 279-285, 2005.

13. Sakaguchi S: Uber eine neue farbenreaktion von protein und arginin. J Biochem 5: 25-31, 1925.

14. Weber CJ: A modification of Sakaguchi's reaction for the quantitative determination of arginine. J Biol Chem 86: 217-222, 1930.

15. Messineo L: Modification of the Sakaguchi reaction: spectrophotometric determination of arginine in proteins without previous hydrolysis. Arch Biochim Biophys 117: 538-540, 1966.

16. Van Pilsum JF, Martin RP, Kito E and Hess J: Determination of creatine, creatinine, arginine, guanidinoacetic acid, guanidine and methyl-guanidine in biological fluids. J Biol Chem 222: 225-236, 1956.

17. Larsson R and Nygren P: A rapid fluorometric method for semiautomated determination of cytotoxicity and cellular proliferation of human tumor cell lines in microculture. Anticancer Res 9: 1111-1119, 1989.
18. Minotti G, Menna P, Salvatorelli E, Cairo G and Gianni L: Anthracyclines: molecular advances and pharmacologic developments in antitumor activity and cardiotoxicity. Pharmacol Rev 56: 185-229, 2004.

19. Maestre N, Tritton TR, Laurent G and Jaffrezou JP: Cell surface-directed interaction of anthracyclines leads to cytotoxicity and nuclear factor $\mathrm{kB}$ activation but not apoptosis signaling. Cancer Res 61: 2558-2561, 2001.

20. Gewirtz DA: A critical evaluation of the mechanisms of action proposed for the antitumor effects of the anthracycline antibiotics adriamycin and daunorubicin. Biochem Pharmacol 57: 727-741, 1999.

21. Di Leo A and Moretti E: Anthracyclines: the first generation of cytotoxic targeted agents? A possible dream. J Clin Oncol 26: 5011-5013, 2008.

22. Kroemer G and Pouyssegur J: Tumor cell metabolism: cancer's Achilles' heel. Cancer Cell 13: 472-482, 2008.

23. Welch JE, Bengtson P, Svensson K, et al: Single chain fragment anti-heparan sulfate antibody targets the polyamine transport system and attenuates polyamine-dependent cell proliferation. Int J Oncol 32: 749-756, 2008

24. Criss WE: A review of polyamines and cancer. Turk J Med Sci 33: 195-205, 2003.

25. Holley JL, Mather A, Wheelhouse RT, Cullis PM, Hartley JA, Bingham JP and Cohen GM: Targeting of tumor cells and DNA by a chlorambucil-spermidine conjugate. Cancer Res 52: 4190-4195, 1992.

26. Zhang Y and Cremer PS: Interactions between macromolecules and ions: the Hofmeister series. Curr Opin Chem Biol 10: 658-663, 2006. 